\title{
ORIGINAL ARTICLE Equivalence of afternoon spot and 24-h urinary hydration biomarkers in free-living healthy adults
}

\author{
JH Bottin, G Lemetais, M Poupin, L Jimenez and ET Perrier
}

BACKGROUND/OBJECTIVES: Urinary biomarkers of hydration (urine osmolality, $U_{\text {Osm; }}$ urine specific gravity, $U_{\mathrm{SG}}$ ) follow circadian variations. For individuals, researchers and health-care professionals, there is value in identifying time frames during which spot values of $U_{\text {Osm }}$ and $U_{S G}$ are representative of 24 -h values in healthy young adults.

SUBJECTS/METHODS: Eighty-two free-living adults $\left(22.3 \pm 2.9\right.$ years, $\left.22.2 \pm 1.5 \mathrm{~kg} / \mathrm{m}^{2}\right)$ collected individual urine voids over a $24-\mathrm{h}$ period. $U_{\text {Osm }}$ and $U_{S G}$ were measured on each void and on the pooled 24-h sample. To determine the time of day when a spot sample was likely to be equivalent to the 24-h value, daytime voids were binned by time and equivalence was tested for each $2-\mathrm{h}$ window. Equivalence was a priori defined as being within $100 \mathrm{mOsm} / \mathrm{kg}\left(U_{\mathrm{Osm}}\right)$ and within 0.003 units $\left(U_{\mathrm{SG}}\right)$ of $24-\mathrm{h}$ values.

RESULTS: For both $U_{\mathrm{Osm}}$ and $U_{\mathrm{SG}}$, voids between 1400 and 2000 hours produced values that were equivalent to the 24-h sample,

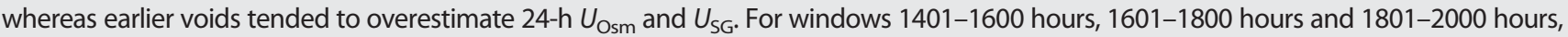
the mean difference (95\% confidence interval) between spot and 24-h $U_{\text {Osm }}(\mathrm{mOsm} / \mathrm{kg})$ was $-25(-72 ; 22), 28(-35 ; 92)$ and $12(-41 ; 66)$, respectively, whereas for $U_{\mathrm{SG}}$ the difference was $0.0014(-0.0028 ;-0.0001), 0.0001(-0.0017 ; 0.0019)$ and 0.0005 $(-0.0018 ; 0.0009)$, respectively.

CONCLUSIONS: In free-living healthy French adults, 24-h urine concentration can be approximated from a mid- to late-afternoon spot urine sample. This finding suggests that an afternoon sample may be an accurate and practical tool for hydration monitoring, useful to individuals and health-care practitioners.

European Journal of Clinical Nutrition (2016) 70, 904-907; doi:10.1038/ejcn.2015.217; published online 13 January 2016

\section{INTRODUCTION}

Water is an essential nutrient to human life, and it enables numerous functions in the body as a carrier, solvent, thermoregulator and reactant among others. As body water is lost daily through urine and stool, perspiration and respiration, replenishment assured by fluid and food intake is necessary and is crucial to maintain optimal hydration. However, water needs vary considerably between individuals depending on factors such as body size, activity levels, dietary habits and climate. Recent studies have highlighted that low daily fluid intake or hypohydration may be associated with detrimental health outcomes such as increased risk of chronic kidney disease, ${ }^{1}$ lithiasis $^{2}$ and impaired glucose homeostasis. ${ }^{3}$ Thus, evaluating hydration at the individual level is clinically relevant for disease risk prevention.

Urinary biomarkers such as 24 -h urine osmolality $\left(U_{\text {Osm }}\right)$ and urine specific gravity $\left(U_{S G}\right)$ have been validated as accurate markers of habitual total fluid intake (TFI) in the general population. ${ }^{4}$ In healthy individuals, these biomarkers can discriminate euhydration from hypohydration, ${ }^{5,6}$ and they are responsive to changes in $\mathrm{TFI}^{7}{ }^{7}$ In patients, high urine concentration has been associated with an increased risk of renal crystal formation, ${ }^{8}$ and maintaining a low $U_{\mathrm{SG}}(<1.010)$ has been recommended as a general preventive measure for kidney stone disease. ${ }^{9}$ Thus, biomarkers of urine concentration are highly useful for daily hydration monitoring, and they may be of clinical relevance for the evaluation of health-associated risks.

Urine concentration is subject to within-day fluctuations, and urinary biomarkers of hydration demonstrate circadian variation. ${ }^{7}$ Previous research has shown that morning voids tend to overestimate 24-h values. ${ }^{7,10}$ This is unsurprising, as firstmorning urine represents the first void after a period of fasting (that is, during sleep). In fact, a concentrated first-morning urine sample represents normal functioning of the kidney, as nighttime antidiuresis allows individuals to sleep through the night. However, the equivalence between spot and 24-h urine concentration has not been properly evaluated at other times of the day. Previously, we determined that a pooled urine sample of afternoon urine was a better candidate for approximating 24-h urine concentration. ${ }^{7}$ However, this previous study was performed in inpatient conditions during which the time and dose of water intake was tightly controlled. Thus, we sought to build upon these results by evaluating the equivalence of spot urine concentration to the $24-h$ value in free-living conditions.

The primary purpose of this study was to identify the time frames during which spot and corresponding 24-h urine concentration ( $U_{\mathrm{Osm}}$ and $\left.U_{\mathrm{SG}}\right)$ were equivalent in free-living healthy young adults from the general population with a wide range of fluid intake. We hypothesized that afternoon, but not morning or evening, spot urine concentration would be equivalent to $24-\mathrm{h}$ values.

\section{MATERIALS AND METHODS}

\section{Participant recruitment}

This prospective cross-sectional study was conducted at a single investigating center. Participants were eligible if they were 20-30 years old, in good health, as determined by medical history, physical examination and hematology and biochemistry screening, and with a body mass index between 20 and $25 \mathrm{~kg} / \mathrm{m}^{2}$. Female subjects were

Danone Research, Palaiseau, France. Correspondence: Dr ET Perrier, Danone Research, RD 128, Palaiseau 91767, France. 
included only if they were on a monophasic oral contraceptive method and not pregnant or breast-feeding. Exclusion criteria included smoking $>10$ cigarettes/day, excessive alcohol consumption ( $>20 \mathrm{~g}$ alcohol/day), use of any prescription or over-the-counter medication that may interfere with water balance or metabolism within 14 days before the start of the study, any clinically relevant acute or chronic diseases and following a vegetarian diet. All participants gave written informed consent before the start of the study. The study was conducted in accordance with the declaration of Helsinki ethical principles, it was approved by an Independent Ethics Committee (Comité de Protection des Personnes EstIII, Nancy, France) and it was registered on clinicaltrials.gov (NCT02044679). Subjects were asked to refrain from intensive physical activity that would result in substantial sweat loss during the study period.

\section{Screening phase}

To ensure a wide representation of daily TFI and thus a range of urine output, potential participants completed a 3-day screening phase during which they recorded all daily food and fluid intake (NutriSaas24WQ-waters; MXS, Paris, France). Participants were included if their mean daily TFI was between 50 and $200 \%$ of the dietary reference value (DRV) determined by the European Food Safety Authority (EFSA). Participants were stratified into three predefined arms: ARM 1, consuming between 50 and $80 \%$ of the EFSA DRV; ARM 2 (TFI $81-120 \%$ of EFSA DRV); and ARM 3 (TFI between 121 and $200 \%$ of EFSA DRV). As TFI in a population is generally relatively normally distributed, these arms were used to ensure adequate representation of 'low' and 'high' drinkers, which would otherwise be underrepresented relative to 'medium' drinkers in a sample. The required total sample size was calculated from previously published data to obtain a power of $90 \%$, and a risk of $5 \%$. A total of 63 participants were required, but ultimately 82 participants were recruited (ARM 1, $n=32$; ARM 2, $n=28$; and ARM 3, $n=22$ ) in order to account for anticipated withdrawal.

\section{Data collection}

The week following the screening phase, participants were asked to maintain their normal daily eating and drinking habits and to record their food and fluid intake on a web-based food and fluid questionnaire (NutriSaas-24WQ-waters; MXS) during three consecutive weekdays. On the third day, they collected individual voids over a full 24-h period. Subjects woke up before 0700 hours, voided and discarded this firstmorning sample. All subsequent voids were collected in individual containers, including the first-morning void of the following morning at 0700, thus completing the 24-h sample. For each void, participants indicated the time of collection. Urine samples were returned to the study center immediately after the final void collection. $U_{S G}$ (Pen Urine S.G., Atago, Tokyo, Japan) and $U_{\text {Osm }}$ (Advanced Model 2020 Multi-Sample Osmometer; Advanced Instruments, Inc., Norwood, MA, USA) were measured on each void, after which all voids were pooled, and measures were repeated on the resulting 24 -h sample.

\section{Statistical method: assessment of equivalence}

The analysis of equivalence was restricted to daytime voids because previous research has demonstrated that early morning and overnight samples tend to be more concentrated than corresponding 24-h samples. ${ }^{7}$ Samples were binned by the time of the void (1000-1200 hours; 1201-1400 hours; $1401-1600$ hours; $1601-1800$ hours; and 1801-2000 hours). If a participant voided more than once in any 2 -h window, the second void was discarded from the equivalence analysis to avoid unequally weighting data from subjects who voided more frequently. As all subjects did not void within every 2 -h window, the number of samples analyzed in each bin could be lower than the total number of subjects. However, each bin included samples from ARM 1, ARM 2 and ARM 3, as planned by the stratified recruitment to ensure a range of urine concentration measurements.

Spot and 24-h $U_{\mathrm{Osm}}$ were considered to be equivalent if the mean difference and $95 \%$ confidence interval $(\mathrm{Cl})$ of the difference fell within $\pm 100 \mathrm{mOsm} / \mathrm{kg}$ (bounds for equivalence set a priori). Although there is no published standard for the bounds of equivalence between 24-h and spot $U_{\text {Osm }}$ our aim was to establish a narrow enough window of $U_{\text {Osm }}$ that would retain enough discriminating ability to differentiate individuals with differences in daily fluid intake. ${ }^{11}$ We also considered the normal physiological range of $U_{\mathrm{Osm}}{ }^{10,12}$ which we approximated as falling between roughly 200 and $1200 \mathrm{mOsm} / \mathrm{kg}$. Upon evaluating these sources, we considered that $\pm 100 \mathrm{mOsm} / \mathrm{kg}$, representing $10 \%$ of the normal range of urine concentration, was sufficiently precise to ensure discrimination between low and high drinkers.

Equivalence was assessed using the two one-sided test approach, which uses two one-sided $t$-tests applied to the a priori established interval for equivalence $\left(U_{\mathrm{Osm}} \pm 100 \mathrm{mOsm} / \mathrm{kg}\right)$. Essentially, the two one-sided test method tests two null hypotheses that the mean difference is (a) not as large as the upper bound, nor (b) below the lower bound a priori established as practically equivalent. For the test (spot) sample to be considered practically equivalent to the reference (24-h sample), both null hypotheses must be rejected, and thus the traditional alpha $(P=0.05)$ reduced by half $(P=0.025)$ for each test.

Spot and 24-h $U_{S G}$ equivalence was analyzed secondarily. Spot and 24-h $U_{S G}$ were considered to be equivalent if the mean difference and $95 \% \mathrm{Cl}$ of the difference fell within \pm 0.003 units of the 24 -h value.

\section{RESULTS}

Total fluid intake

Mean daily TFI from the participants who completed the study ( $n=82$, age: $22.3 \pm 2.9$ years, body mass index: $22.2 \pm 1.5 \mathrm{~kg} / \mathrm{m}^{2}, 41$ females) ranged from 0.69 to $4.35 \mathrm{l} /$ day with a mean of $1.83 \pm 0.65 \mathrm{l} /$ day (Table 1). As planned, there were differences in mean TFI between the 3 arms. The number of samples analyzed in each bin is presented in Table 2 .

Equivalence of Spot and 24-h $U_{\text {Osm }}$

Mean (s.d.) 24-h $U_{\text {Osm }}$ was 567 (214) mOsm/kg, ranging from 149 to $1029 \mathrm{mOsm} / \mathrm{kg}$. Equivalence (defined as a difference of $<100 \mathrm{mOsm} / \mathrm{kg}$ from the 24-h value) was demonstrated for afternoon urine samples (from 1401 to 2000 hours), whereas morning and midday samples (1000-1400 hours) tended to overestimate 24-h $U_{\text {Osm }}$. The distribution of the absolute difference $(95 \% \mathrm{Cl})$ between spot and 24-h $U_{\mathrm{Osm}}$ values for each 2 -h bin is shown in Table 3. The mean differences ( $95 \% \mathrm{Cl} ; \mathrm{mOsm} / \mathrm{kg}$ ) from the 24 -h sample were 83 (12 to 154 ) from $1000-1200$ hours; 107 (58 to 157) from $1201-1400$ hours; -25 ( -72 to 22 ) from 1401-1600 hours; 28 (-35 to 92) from $1601-1800$ hours; and 12 (-41 to 66) from $1801-2000$ hours.

Table 1. Demographic characteristics and total fluid intake of participants

\begin{tabular}{lcccc}
\hline & ARM 1 & \multicolumn{1}{c}{ ARM 2 } & \multicolumn{1}{c}{ ARM 3 } & Total \\
\hline $\mathrm{N}(\mathrm{F} / \mathrm{M})$ & $32(12 / 20)$ & $28(15 / 13)$ & $22(14 / 8)$ & $82(41 / 41)$ \\
Age (s.d.) in years & $23.6(3.1)$ & $23.3(2.8)$ & $23.9(2.8)$ & $23.6(2.9)$ \\
BMI (s.d.) in kg/m & $22.0(1.6)$ & $22.0(1.6)$ & $22.4(1.4)$ & $22.1(1.6)$ \\
TFI (ml/day) & $1430(490)$ & $1825(405)$ & $2414(671)$ & $1830(648)$ \\
\hline
\end{tabular}

Abbreviations: $\mathrm{BMI}$, body mass index; $\mathrm{F}$, female; $\mathrm{M}$, male; $\mathrm{TFI}$, total fluid intake.

Table 2. Number of individual voids included per 2-h assessment window, stratified by TFI

\begin{tabular}{|ccccc|}
\hline Time of void (hours) & \multicolumn{5}{c}{ Number of samples per arm $\mathrm{n}(\%)$} \\
\cline { 2 - 5 } & $\begin{array}{c}\text { ARM 1 } \\
(\mathrm{n}=32)\end{array}$ & $\begin{array}{c}\text { ARM 2 } \\
(\mathrm{n}=28)\end{array}$ & $\begin{array}{c}\text { ARM 3 } \\
(\mathrm{n}=22)\end{array}$ & $\begin{array}{c}\text { Total } \\
(\mathrm{n}=82)\end{array}$ \\
\hline $1000-1200$ & $15(47 \%)$ & $11(39 \%)$ & $14(64 \%)$ & $40(49 \%)$ \\
$1201-1400$ & $23(72 \%)$ & $19(68 \%)$ & $16(73 \%)$ & $58(71 \%)$ \\
$1401-1600$ & $18(56 \%)$ & $15(54 \%)$ & $9(41 \%)$ & $42(51 \%)$ \\
$1601-1800$ & $14(44 \%)$ & $14(50 \%)$ & $16(73 \%)$ & $44(54 \%)$ \\
$1801-2000$ & $20(63 \%)$ & $20(71 \%)$ & $12(55 \%)$ & $52(63 \%)$ \\
\hline
\end{tabular}


Table 3. Mean absolute difference between spot and 24-h $U_{\text {Osm }}$

\begin{tabular}{|c|c|c|c|c|}
\hline \multirow[t]{2}{*}{ Time window (hours) } & \multirow{2}{*}{$\begin{array}{l}\text { Mean absolute difference } \\
\text { between spot and 24-h } \bigcup_{\text {Osm }}\end{array}$} & \multicolumn{2}{|c|}{$95 \% \mathrm{Cl}$ of mean $(\mathrm{mOsm} / \mathrm{kg})$} & \multirow[t]{2}{*}{ Interpretation } \\
\hline & & Lower limit & Upper limit & \\
\hline $1201-1400$ & 107.2 & 57.60 & 156.74 & Not equivalent \\
\hline $1401-1600$ & -24.9 & -72.19 & 22.48 & Equivalent \\
\hline $1601-1800$ & 28.5 & -35.25 & 92.25 & Equivalent \\
\hline $1801-2000$ & 12.4 & -40.97 & 65.78 & Equivalent \\
\hline
\end{tabular}

Equivalence of spot and 24-h $U_{\mathrm{SG}}$

Mean (s.d.) 24-h $U_{\mathrm{SG}}$ was 1.015 (0.005), ranging between 1.000 and 1.030. Consistent with $U_{\text {Osm }}$ equivalence, spot $U_{S G}$ was equivalent to $24-\mathrm{h} U_{\mathrm{SG}}$ (mean difference of $<0.003$ units from the 24-h value) during the same afternoon bins (1401-2000 hours) and overestimated in the morning and midday voids (1000-1400 hours). Specifically, the mean differences (95\% Cl) between 24-h and spot $U_{S G}$ were $0.0024(0.0004$ to 0.0043$)$ at $1000-1200$ hours (not equivalent); 0.0019 (0.0006 to 0.0032$)$ at $1201-1400$ hours (not equivalent); 0.0014 ( -0.0028 to -0.0001$)$ at $1401-1600$ hours (equivalent); $0.0001(-0.0017$ to 0.0019$)$ at $1601-1800$ hours (equivalent); and 0.0005 (-0.0018 to 0.0009$)$ at 1801-2000 hours (equivalent).

Correlation between $U_{\text {Osm }}$ and $U_{\text {SG }}$

$U_{\text {Osm }}$ and $U_{\text {SG }}$ were very highly correlated $\left(r^{2}=0.9243\right)$, as shown in Figure 1.

\section{DISCUSSION}

Low water intake or low 24-h urine volume has been associated with detrimental health outcomes such as increased risk of chronic kidney disease, incidence of kidney stone formation and impaired glucose homeostasis. ${ }^{1-3}$ However, neither fluid intake volume nor urine volume alone can adequately take into account differences in individual water requirements because of physical activity and climate, dietary solute load or other factors. Measures of urine concentration may be better-suited to help individuals determine the adequacy of their hydration behaviors. Indeed, targets have been proposed for $24-\mathrm{h} U_{\mathrm{Osm}}{ }^{13} U_{\mathrm{SG}}{ }^{9}$ and free-water reserve. ${ }^{14}$ However, 24-h urine collections are burdensome and inconvenient for individual hydration monitoring, and it is well documented that first-morning voids, although often used in population sampling and health-care settings, tend to overestimate 24-h urine concentration. ${ }^{7,10}$ Thus, the aim of this study was to identify time frames during which spot and 24-h values of $U_{\text {Osm }}$ and $U_{\text {SG }}$ were equivalent to the 24 -h sample in healthy adults in the general population.

Using relatively tight bounds, $U_{\mathrm{Osm}}$ and $U_{\mathrm{SG}}$ in spot urine samples obtained in the afternoon and early evening (14012000 hours) were determined to be equivalent to corresponding $24-h$ values. Our results confirm and build upon previous research ${ }^{7}$ in an inpatient setting with standardized water intake, meals and sleep/wake hours showing that $24-\mathrm{h} U_{\text {Osm }}$ is overestimated in morning and late evening collections. Moreover, the previous study collected all voids produced during a given time frame in the same collection container; in contrast, by using single spot urine samples, the current study gives a degree of precision into the timing of a spot urine sample that was not possible in the previous work. Overall, the current findings extend the external validity of previous work, demonstrating the utility of afternoon spot urine across a range of habitual, ad-libitum drinking

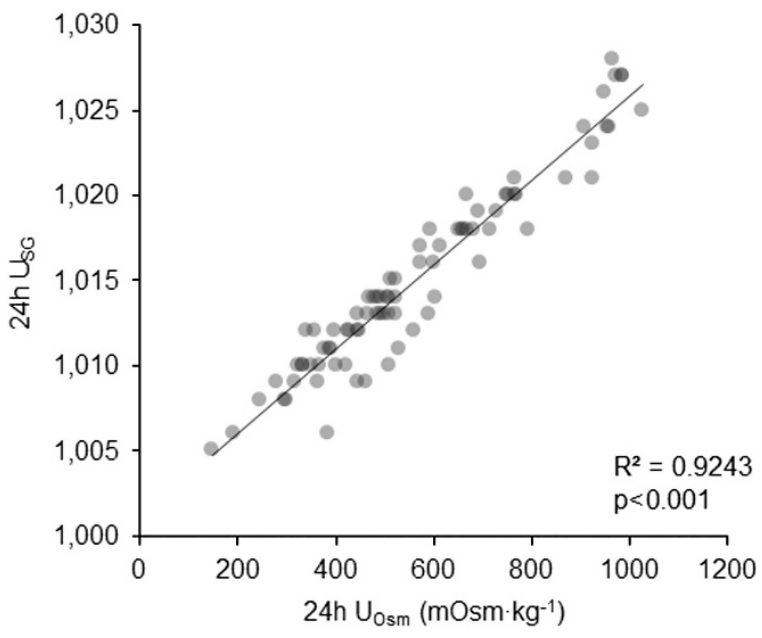

Figure 1. Correlation between 24-h $U_{\text {Osm }}$ and 24-h $U_{\text {SG. }}$.

behaviors. This is of particular relevance because previous research has shown large variability in TFI both between and within countries worldwide. ${ }^{15}$ For studies in which a 24 -h urine collection is not feasible, an afternoon spot sample may reduce the burden on patients and clinicians, and improve the likelihood that clinicians may include measure of spot urine concentration as part of routine patient exams.

In line with the results of previous studies, ${ }^{7,10}$ we showed that morning urine concentration was significantly elevated compared with corresponding 24-h values. This is a logical finding, as the first-morning void represents the kidney's normal nighttime antidiuretic activity, allowing for water conservation and uninterrupted sleep. However, this suggests that in studies using first-morning osmolality as a marker of hydration the prevalence of 24-h hypohydration may be overestimated. Future work should focus on evaluating whether urinary biomarkers of hydration in first-morning samples can be corrected to accurately represent 24-h values, especially in large observational cohorts in which 24-h or afternoon urine collections are impossible.

$U_{\text {Osm }}$ has a high degree of measurement resolution, but it is both costly and time-consuming to measure. For this reason, it is seldom used by health-care practitioners and inaccessible to individuals from the general population. $U_{S G}$ requires less equipment and technical expertise. To simplify daily hydration monitoring in free-living adults, $U_{\mathrm{SG}}$ in afternoon spot samples appears to be a practical, inexpensive and a simple alternative. It is important to consider, however, that this study included only young healthy adults with no known kidney disorders. The confirmation of equivalence between afternoon spot and 24-h values of urine concentration in those populations would be of significant importance for the risk assessment of chronic kidney disease and kidney stones. 
One of the strengths of our study is its stratified design that ensured a wide range of urine concentration, and the outpatient setting in which participants' food and fluid consumption habits (timing and volumes) were representative of normal daily habits. This contributes to the external validity of the findings. This study also has several limitations. It was carried out in a population of French adults, who typically consume three main meals per day, breakfast, lunch and dinner-a pattern reflective of many western cultures. It is known that meal times, composition and beverage consumption are influenced by many cultural, ethnic, educational and geographical factors. The results from the current study may therefore only be generalized to the extent to which other cultures follow similar eating and drinking patterns. Importantly, equivalence was demonstrated for samples obtained after the usual midday meal time, but not for samples obtained before the midday meal. The results may not apply to cultures in which meal times and eating patterns are substantially different from this structure, as well as to individuals whose schedules are offset from normal sleeping and waking hours, such as night-shift workers or individuals whose circadian rhythms are continuously being challenged. Finally, to our knowledge, no bounds for equivalence of hydration biomarkers have been published and validated. We therefore recognize that the bounds for equivalence used in this analysis were set a priori according to assumptions made from previous research.

\section{CONCLUSION}

$U_{\text {Osm }}$ and $U_{S G}$ in spot urine samples obtained in the afternoon and early evening (1401-2000 hours) were equivalent to corresponding $24-h$ values in free-living healthy young adults and may therefore represent a convenient alternative to 24-h urine samples to monitor daily hydration in real-life conditions. In particular, $U_{\mathrm{SG}}$ measured in afternoon spot samples appears to be a practical, inexpensive and a simple tool to monitor daily hydration.

\section{CONFLICT OF INTEREST}

JHB, GL, MP, LJ and ETP are employees of Danone Research, France.

\section{ACKNOWLEDGEMENTS}

This study was funded by Danone Research.

\section{AUTHOR CONTRIBUTION}

All authors have read and approved the final paper.

\section{REFERENCES}

1 Strippoli GF, Craig JC, Rochtchina E, Flood VM, Wang JJ, Mitchell P. Fluid and nutrient intake and risk of chronic kidney disease. Nephrology (Carlton) 2011; 16: 326-334.

2 Sorensen MD, Kahn AJ, Reiner AP, Tseng TY, Shikany JM, Wallace RB et al. Impact of nutritional factors on incident kidney stone formation: a report from the WHI OS. J Urol 2012; 187: 1645-1649.

3 Roussel R, Fezeu L, Bouby N, Balkau B, Lantieri O, Alhenc-Gelas F et al. Low water intake and risk for new-onset hyperglycemia. Diabetes Care 2011; 34: 2551-2554.

4 Perrier E, Rondeau P, Poupin M, Le BL, Armstrong LE, Lang F et al. Relation between urinary hydration biomarkers and total fluid intake in healthy adults. Eur J Clin Nutr 2013; 67: 939-943.

5 Armstrong LE, Maresh CM, Castellani JW, Bergeron MF, Kenefick RW, LaGasse KE et al. Urinary indices of hydration status. Int J Sport Nutr 1994; 4: 265-279.

6 Armstrong LE, Soto JA, Hacker Jr FT, Casa DJ, Kavouras SA, Maresh CM. Urinary indices during dehydration, exercise, and rehydration. Int J Sport Nutr 1998; 8: 345-355.

7 Perrier E, Demazieres A, Girard N, Pross N, Osbild D, Metzger D et al. Circadian variation and responsiveness of hydration biomarkers to changes in daily water intake. Eur J Appl Physiol 2013; 113: 2143-2151.

8 Hennequin C, Daudon M, Phung T, Lacour B, Jungers P. Evaluation of the lithogenic risk in renal lithiasis. Value of urine density measurement. Presse Med 1995; 24: 1559-1562.

9 European Association of Urology, Türk C, Knoll T, Petrik A, Sarica K, Skolarikos A et al. Guidelines on Urolithiasis, 2015. Available at http://uroweb.org/wp-content/ uploads/22-Urolithiasis_LR.pdf.

10 Armstrong LE, Pumerantz AC, Fiala KA, Roti MW, Kavouras SA, Casa DJ et al. Human hydration indices: acute and longitudinal reference values. Int J Sport Nutr Exerc Metab 2010; 20: 145-153.

11 Perrier E, Vergne S, Klein A, Poupin M, Rondeau P, Le BL et al. Hydration biomarkers in free-living adults with different levels of habitual fluid consumption. Br J Nutr 2013; 109: 1678-1687.

12 Armstrong LE, Johnson EC, Munoz CX, Swokla B, Le Bellego L, Jimenez L et al. Hydration biomarkers and dietary fluid consumption of women. $J$ Acad Nutr Diet 2012: 112: 1056-1061.

13 Perrier ET, Buendia-Jimenez I, Vecchio M, Armstrong LE, Tack I, Klein A. Twenty-four-hour urine osmolality as a physiological index of adequate water intake. Dis Markers 2015; 2015: 231063.

14 Manz F, Johner SA, Wentz A, Boeing H, Remer T. Water balance throughout the adult life span in a German population. Br J Nutr 2012; 107: 1673-1681.

15 Guelinckx I, Ferreira-Pego C, Moreno LA, Kavouras SA, Gandy J, Martinez $\mathrm{H}$ et al. Intake of water and different beverages in adults across 13 countries. Eur J Nutr 2015; 54: 45-55.
(C) $(-)$ This work is licensed under a Creative Commons AttributionBY NC ND NonCommercial-NoDerivs 4.0 International License. The images or other third party material in this article are included in the article's Creative Commons license, unless indicated otherwise in the credit line; if the material is not included under the Creative Commons license, users will need to obtain permission from the license holder to reproduce the material. To view a copy of this license, visit http:// creativecommons.org/licenses/by-nc-nd/4.0/ 